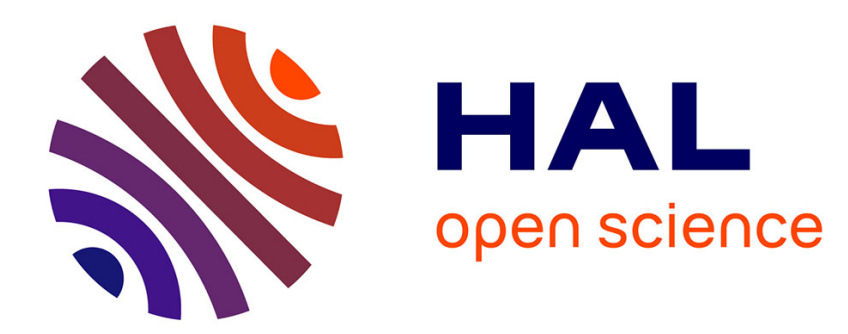

\title{
Ocular symptoms are not predictive of ophthalmologic inflammation in inflammatory bowel disease
}

Véronique Cloché

\section{To cite this version:}

Véronique Cloché. Ocular symptoms are not predictive of ophthalmologic inflammation in inflammatory bowel disease. Life Sciences [q-bio]. 2012. hal-01739166

\section{HAL Id: hal-01739166 \\ https://hal.univ-lorraine.fr/hal-01739166}

Submitted on 20 Mar 2018

HAL is a multi-disciplinary open access archive for the deposit and dissemination of scientific research documents, whether they are published or not. The documents may come from teaching and research institutions in France or abroad, or from public or private research centers.
L'archive ouverte pluridisciplinaire HAL, est destinée au dépôt et à la diffusion de documents scientifiques de niveau recherche, publiés ou non, émanant des établissements d'enseignement et de recherche français ou étrangers, des laboratoires publics ou privés. 


\section{(1) UNIVERSITÉ}

\section{AVERTISSEMENT}

Ce document est le fruit d'un long travail approuvé par le jury de soutenance et mis à disposition de l'ensemble de la communauté universitaire élargie.

II est soumis à la propriété intellectuelle de l'auteur. Ceci implique une obligation de citation et de référencement lors de l'utilisation de ce document.

D'autre part, toute contrefaçon, plagiat, reproduction illicite encourt une poursuite pénale.

Contact : ddoc-thesesexercice-contact@univ-lorraine.fr

\section{LIENS}

Code de la Propriété Intellectuelle. articles L 122. 4

Code de la Propriété Intellectuelle. articles L 335.2- L 335.10

http://www.cfcopies.com/V2/leg/leg_droi.php

http://www.culture.gouv.fr/culture/infos-pratiques/droits/protection.htm 


\section{THÈSE}

pour obtenir le grade de

\section{DOCTEUR EN MÉDECINE}

Présentée et soutenue publiquement dans le cadre du troisième cycle de Médecine Spécialisée

\section{Véronique CLOCHÉ}

le $29 / 06 / 2012$

Les symptômes oculaires sont-ils prédictifs d'une inflammation ophtalmologique au cours des maladies inflammatoires chroniques intestinales?

Etude prospective chez 306 patients.

Examinateurs de la thèse :

Mme le Professeur K. ANGIOI-DUPREZ

Président

M. le Professeur JL. GEORGE

Juge

M. le Professeur JP. BERROD

Juge

M. le Professeur L. PEYRIN BIROULET

Juge 


\title{
UNIVERSITÉ DE LORRAINE
}

\section{FACULTÉ DE MÉDECINE DE NANCY}

\section{Président de l'Université de Lorraine : Professeur Pierre MUTZENHARDT \\ Doyen de la Faculté de Médecine : Professeur Henry COUDANE}

\author{
Vice Doyen « Pédagogie » : Professeur Karine ANGIOI \\ Vice Doyen Mission "sillon lorrain » : Professeur Annick BARBAUD \\ Vice Doyen Mission "Campus " : Professeur Marie-Christine BÉNÉ \\ Vice Doyen Mission « Finances » : Professeur Marc BRAUN \\ Vice Doyen Mission « Recherche » : Professeur Jean-Louis GUÉANT
}

Assesseurs :

-1 Cycle:

Professeur Bruno CHENUEL

- « Première année commune aux études de santé (PACES) et M. Christophe NEMOS

universitarisation études para-médicales ॥

$-2^{\text {eme }}$ Cycle

- ${ }^{\text {eme }}$ Cycle:

"DES Spécialités Médicales, Chirurgicales et Biologiques »

«DES Spécialité Médecine Générale

- Filières professionnalisées:

- Formation Continue :

- Commission de Prospective :

- Recherche:

- Développement Professionnel Continu:

Assesseurs Relations Internationales

Professeur Marc DEBOUVERIE

Professeur Jean-Pierre BRONOWICKI Professeur Francis RAPHAËL

M. Walter BLONDEL

Professeur Hervé VESPIGNANI

Professeur Pierre-Edouard BOLLAERT

Professeur Didier MAINARD

Professeur Jean-Dominique DE KORWIIN

Professeur Jacques HUBERT

\section{DOYENS HONORAIRES}

Professeur Adrien DUPREZ - Professeur Jean-Bernard DUREUX

Professeur Jacques ROLAND - Professeur Patrick NETTER

ニニニニニニニニニニ

\section{PROFESSEURS HONORAIRES}

Pierre ALEXANDRE - Jean-Marie ANDRE - Daniel ANTHOINE - Alain BERTRAND - Pierre BEY - Patrick BOISSEL Jacques BORRELLY - Michel BOULANGE - Jean-Claude BURDIN - Claude BURLET - Daniel BURNEL

Claude CHARDOT - Jean-Pierre CRANCE - Gérard DEBRY - Jean-Pierre DELAGOUTTE - Emile de LAVERGNE Jean-Pierre DESCHAMPS - Jean DUHEILLE - Adrien DUPREZ - Jean-Bernard DUREUX - Gérard FIEVE Jean

FLOQUET - Robert FRISCH - Alain GAUCHER - Pierre GAUCHER - Hubert GERARD

Jean-Marie GILGENKRANTZ - Simone GILGENKRANTZ - Oliéro GUERCI - Pierre HARTEMANN - Claude HURIET

Christian JANOT - Michèle KESSLER - Jacques LACOSTE - Henri LAMBERT - Pierre LANDES

Marie-Claire LAXENAIRE - Michel LAXENAIRE - Jacques LECLERE - Pierre LEDERLIN - Bernard LEGRAS

Michel MANCIAUX - Jean-Pierre MALLIÉ - Philippe MANGIN - Pierre MATHIEU - Denise MONERET-VAUTRIN

Pierre MONIN - Pierre NABET - Jean-Pierre NICOLAS - Pierre PAYSANT - Francis PENIN - Gilbert PERCEBOIS

Claude PERRIN - Guy PETIET - Luc PICARD - Michel PIERSON - Jean-Marie POLU - Jacques POUREL

Jean PREVOT - Antoine RASPILLER - Michel RENARD - Jacques ROLAND - René-Jean ROYER - Daniel SCHMITT Michel SCHWEITZER - Claude SIMON - Danièle SOMMELET - Jean-François STOLTZ

Michel STRICKER - Gilbert THIBAUT - Augusta TREHEUX - Hubert UFFHOLTZ - Gérard VAILLANT - Paul VERT Colette VIDAILHET - Michel VIDAILHET - Michel WAYOFF - Michel WEBER

ニニニニニニニニニニ

\section{PROFESSEURS DES UNIVERSITÉS PRATICIENS HOSPITALIERS}

(Disciplines du Conseil National des Universités)

\author{
$42^{\text {ème }}$ Section : MORPHOLOGIE ET MORPHOGENÈSE \\ 1ère sous-section : (Anatomie) \\ Professeur Gilles GROSDIDIER \\ Professeur Pierre LASCOMBES - Professeur Marc BRAUN
}




$$
\begin{gathered}
2^{\text {ème }} \text { sous-section : (Cytologie et histologie) } \\
\text { Professeur Bernard FOLIGUET } \\
3^{\text {ème }} \text { sous-section : (Anatomie et cytologie pathologiques) } \\
\text { Professeur François PLENAT - Professeur Jean-Michel VIGNAUD }
\end{gathered}
$$

$43^{\text {ème }}$ Section : BIOPHYSIQUE ET IMAGERIE MÉDICALE

$1^{\text {ère }}$ sous-section : (Biophysique et médecine nucléaire)

Professeur Gilles KARCHER - Professeur Pierre-Yves MARIE - Professeur Pierre OLIVIER $2^{\mathrm{e} m e}$ sous-section : (Radiologie et imagerie médicale)

Professeur Denis REGENT - Professeur Michel CLAUDON - Professeur Valérie CROISÉ-LAURENT

Professeur Serge BRACARD - Professeur Alain BLUM - Professeur Jacques FELBLINGER Professeur René ANXIONNAT

$44^{\text {ème }}$ Section : BIOCHIMIE, BIOLOGIE CELLULAIRE ET MOLÉCULAIRE, PHYSIOLOGIE ET NUTRITION $1^{\text {ère }}$ sous-section : (Biochimie et biologie moléculaire)

Professeur Jean-Louis GUÉANT - Professeur Jean-Luc OLIVIER - Professeur Bernard NAMOUR $2^{\text {ème }}$ sous-section : (Physiologie)

Professeur François MARCHAL - Professeur Bruno CHENUEL - Professeur Christian BEYAERT $3^{\text {ème }}$ sous-section : (Biologie Cellulaire)

$$
\begin{aligned}
& \text { Professeur Ali DALLOUL } \\
& 4^{\text {eme }} \text { sous-section : (Nutrition) }
\end{aligned}
$$

Professeur Olivier ZIEGLER - Professeur Didier QUILLIOT - Professeur Rosa-Maria RODRIGUEZ-GUEANT

$45^{\text {ème }}$ Section : MICROBIOLOGIE, MALADIES TRANSMISSIBLES ET HYGIÈNE

$1^{\text {ère }}$ sous-section : (Bactériologie - virologie; hygiène hospitalière)

Professeur Alain LE FAOU - Professeur Alain LOZNIEWSKI

$3^{\text {ème }}$ sous-section : (Maladies infectieuses; maladies tropicales)

Professeur Thierry MAY - Professeur Christian RABAUD

$46^{\text {ème }}$ Section : SANTÉ PUBLIQUE, ENVIRONNEMENT ET SOCIÉTÉ

$1^{\text {ère }}$ sous-section : (Épidémiologie, économie de la santé et prévention)

Professeur Philippe HARTEMANN - Professeur Serge BRIANÇON - Professeur Francis GUILLEMIN

Professeur Denis ZMIROU-NAVIER - Professeur François ALLA $2^{\text {eme }}$ sous-section : (Médecine et santé au travail)

Professeur Christophe PARIS

$3^{{ }^{\mathrm{m}} \mathrm{me}}$ sous-section : (Médecine légale et droit de la santé)

Professeur Henry COUDANE

$4^{\text {ème }}$ sous-section : (Biostatistiques, informatique médicale et technologies de communication)

Professeur François KOHLER - Professeur Éliane ALBUISSON

$47^{\text {ème }}$ Section : CANCÉROLOGIE, GÉNÉTIQUE, HÉMATOLOGIE, IMMUNOLOGIE

$1^{\text {ère }}$ sous-section : (Hématologie ; transfusion)

Professeur Pierre BORDIGONI - Professeur Jean-François STOLTZ - Professeur Pierre FEUGIER

$2^{\mathrm{e} m e}$ sous-section : (Cancérologie ; radiothérapie)

Professeur François GUILLEMIN - Professeur Thierry CONROY

Professeur Didier PEIFFERT - Professeur Frédéric MARCHAL $3^{\text {ème }}$ sous-section : (Immunologie)

Professeur Gilbert FAURE - Professeur Marie-Christine BENE $4^{\mathrm{e}}{ }^{\mathrm{me}}$ sous-section : (Génétique)

Professeur Philippe JONVEAUX - Professeur Bruno LEHEUP

$48^{\text {ème }}$ Section : ANESTHÉSIOLOGIE, RÉANIMATION, MÉDECINE D'URGENCE, PHARMACOLOGIE ET THÉRAPEUTIQUE

$1^{1 \text { ère }}$ sous-section : (Anesthésiologie et réanimation chirurgicale; médecine d'urgence)

Professeur Claude MEISTELMAN - Professeur Hervé BOUAZIZ

Professeur Paul-Michel MERTES - Professeur Gérard AUDIBERT - Professeur Thomas FUCHS-BUDER

$2^{\mathrm{e} \text { èe }}$ sous-section : (Réanimation médicale ; médecine d'urgence)

Professeur Alain GERARD - Professeur Pierre-Édouard BOLLAERT

Professeur Bruno LÉVY - Professeur Sébastien GIBOT

$3^{{ }^{\mathrm{ème}}}$ sous-section : (Pharmacologie fondamentale; pharmacologie clinique ; addictologie)

Professeur Patrick NETTER - Professeur Pierre GILLET

$4^{\text {ème }}$ sous-section : (Thérapeutique ; médecine d'urgence ; addictologie)

Professeur François PAILLE - Professeur Gérard GAY - Professeur Faiez ZANNAD - Professeur Patrick ROSSIGNOL 
$49^{\text {ème }}$ Section : PATHOLOGIE NERVEUSE ET MUSCULAIRE, PATHOLOGIE MENTALE, HANDICAP et RÉÉDUCATION

$1^{\text {êre }}$ sous-section : (Neurologie)

Professeur Gérard BARROCHE - Professeur Hervé VESPIGNANI

Professeur Xavier DUCROCQ - Professeur Marc DEBOUVERIE - Professeur Luc TAILLANDIER

$2^{\text {ème }}$ sous-section : (Neurochirurgie)

Professeur Jean-Claude MARCHAL - Professeur Jean AUQUE - Professeur Olivier KLEIN

Professeur Thierry CIVIT

$3^{\text {ème }}$ sous-section : (Psychiatrie d'adultes; addictologie)

Professeur Jean-Pierre KAHN - Professeur Raymund SCHWAN

$4^{\mathrm{e} m e}$ sous-section : (Pédopsychiatrie; addictologie)

Professeur Daniel SIBERTIN-BLANC - Professeur Bernard KABUTH

$5^{\mathrm{èm}}$ sous-section : (Médecine physique et de réadaptation)

Professeur Jean PAYSANT

$50^{{ }^{\mathrm{ème}}}$ Section : PATHOLOGIE OSTÉO-ARTICULAIRE, DERMATOLOGIE et CHIRURGIE PLASTIQUE

$1^{\text {ère }}$ sous-section : (Rhumatologie)

Professeur Isabelle CHARY-VALCKENAERE - Professeur Damien LOEUILLE

$2^{\text {ème }}$ sous-section : (Chirurgie orthopédique et traumatologique)

Professeur Daniel MOLE - Professeur Didier MAINARD

Professeur François SIRVEAUX - Professeur Laurent GALOIS $3^{\text {ème }}$ sous-section : (Dermato-vénéréologie)

Professeur Jean-Luc SCHMUTZ - Professeur Annick BARBAUD

$4^{\text {ème }}$ sous-section : (Chirurgie plastique, reconstructrice et esthétique ; brûlologie)

Professeur François DAP - Professeur Gilles DAUTEL

$51^{\text {ème }}$ Section : PATHOLOGIE CARDIORESPIRATOIRE et VASCULAIRE

$1^{\text {ère }}$ sous-section : (Pneumologie ; addictologie)

Professeur Yves MARTINET - Professeur Jean-François CHABOT - Professeur Ari CHAOUAT $2^{\mathrm{e} \text { è }}$ sous-section : (Cardiologie)

Professeur Etienne ALIOT - Professeur Yves JUILLIERE - Professeur Nicolas SADOUL

Professeur Christian de CHILLOU

$3^{\text {ème }}$ sous-section : (Chirurgie thoracique et cardiovasculaire)

Professeur Jean-Pierre VILLEMOT

$4^{\text {ème }}$ sous-section : (Chirurgie vasculaire ; médecine vasculaire)

Professeur Denis WAHL - Professeur Sergueï MALIKOV

$52^{\text {ème }}$ Section : MALADIES DES APPAREILS DIGESTIF et URINAIRE

$1^{\text {ère }}$ sous-section : (Gastroentérologie ; hépatologie ; addictologie)

Professeur Marc-André BIGARD - Professeur Jean-Pierre BRONOWICKI - Professeur Laurent PEYRIN-BIROULET $3^{\mathrm{ème}}$ sous-section : (Néphrologie)

Professeur Dominique HESTIN - Professeur Luc FRIMAT

$4^{\text {eme }}$ sous-section : (Urologie)

Professeur Jacques HUBERT - Professeur Pascal ESCHWEGE

$53^{\text {ème }}$ Section : MÉDECINE INTERNE, GÉRIATRIE et CHIRURGIE GÉNÉRALE

$1^{1 \text { èr }}$ sous-section : (Médecine interne; gériatrie et biologie du vieillissement ; médecine générale ; addictologie)

Professeur Jean-Dominique DE KORWIN - Professeur Pierre KAMINSKY

Professeur Athanase BENETOS - Professeur Gisèle KANNY - Professeur Christine PERRET-GUILLAUME

$2^{\text {ème }}$ sous-section : (Chirurgie générale)

Professeur Laurent BRESLER - Professeur Laurent BRUNAUD - Professeur Ahmet AYAV

$54^{\text {ème }}$ Section : DÉVELOPPEMENT ET PATHOLOGIE DE L'ENFANT, GYNÉCOLOGIE-OBSTÉTRIQUE, ENDOCRINOLOGIE ET REPRODUCTION

$1^{\text {ère }}$ sous-section : (Pédiatrie)

Professeur Jean-Michel HASCOET - Professeur Pascal CHASTAGNER

Professeur François FEILLET - Professeur Cyril SCHWEITZER - Professeur Emmanuel RAFFO $2^{\text {ème }}$ sous-section : (Chirurgie infantile)

Professeur Michel SCHMITT - Professeur Pierre JOURNEAU - Professeur Jean-Louis LEMELLE

$3^{\text {ème }}$ sous-section : (Gynécologie-obstétrique ; gynécologie médicale)

Professeur Jean-Louis BOUTROY - Professeur Philippe JUDLIN - Professeur Patricia BARBARINO

$4^{\mathrm{eme}}$ sous-section : (Endocrinologie, diabète et maladies métaboliques; gynécologie médicale)

Professeur Georges WERYHA - Professeur Marc KLEIN - Professeur Bruno GUERCI 
$55^{\text {ème }}$ Section : PATHOLOGIE DE LA TÊTE ET DU COU

$1^{\text {ere }}$ sous-section : (Oto-rhino-laryngologie)

Professeur Roger JANKOWSKI - Professeur Cécile PARIETTI-WINKLER $2^{\text {ème }}$ sous-section : (Ophtalmologie)

Professeur Jean-Luc GEORGE - Professeur Jean-Paul BERROD - Professeur Karine ANGIOI-DUPREZ

$3^{\mathrm{e} m e}$ sous-section : (Chirurgie maxillo-faciale et stomatologie)

Professeur Jean-François CHASSAGNE - Professeur Etienne SIMON

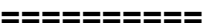

\section{PROFESSEURS DES UNIVERSITÉS}

$64^{\text {ème }}$ Section : BIOCHIMIE ET BIOLOGIE MOLÉCULAIRE

Professeur Sandrine BOSCHI-MULLER

\section{PROFESSEURS DES UNIVERSITÉS DE MÉDECINE GÉNÉRALE} Professeur Jean-Marc BOIVIN

ニニーニニーニニニニ

\section{MAÎTRES DE CONFÉRENCES DES UNIVERSITÉS - PRATICIENS HOSPITALIERS}

$42^{\text {ème }}$ Section : MORPHOLOGIE ET MORPHOGENĖSE

1ère sous-section : (Anatomie)

Docteur Bruno GRIGNON - Docteur Thierry HAUMONT - Docteur Manuela PEREZ

$2^{\text {ème }}$ sous-section : (Cytologie et histologie)

Docteur Edouard BARRAT - Docteur Françoise TOUATI - Docteur Chantal KOHLER

$3^{\text {ème }}$ sous-section : (Anatomie et cytologie pathologiques) Docteur Aude BRESSENOT

$43^{\text {ème }}$ Section : BIOPHYSIQUE ET IMAGERIE MÉDICALE

$1^{1 \text { ère }}$ sous-section : (Biophysique et médecine nucléaire)

Docteur Marie-Hélène LAURENS - Docteur Jean-Claude MAYER

Docteur Pierre THOUVENOT - Docteur Jean-Marie ESCANYE $2^{\text {ème }}$ sous-section : (Radiologie et imagerie médicale) Docteur Damien MANDRY

$44^{\text {ème }}$ Section : BIOCHIMIE, BIOLOGIE CELLULAIRE ET MOLÉCULAIRE, PHYSIOLOGIE ET NUTRITION $1^{\text {ère }}$ sous-section : (Biochimie et biologie moléculaire)

Docteur Jean STRACZEK - Docteur Sophie FREMONT

Docteur Isabelle GASTIN - Docteur Marc MERTEN - Docteur Catherine MALAPLATE-ARMAND Docteur Shyue-Fang BATTAGLIA $3^{\text {ème }}$ sous-section : (Biologie Cellulaire)

Docteur Véronique DECOT-MAILLERET

$45^{\text {ème }}$ Section : MICROBIOLOGIE, MALADIES TRANSMISSIBLES ET HYGIÈNE $1^{\text {ere }}$ sous-section : (Bactériologie - Virologie; hygiène hospitalière)

Docteur Francine MORY - Docteur Véronique VENARD - Docteur Hélène JEULIN $2^{\text {ème }}$ sous-section : (Parasitologie et mycologie)

Docteur Nelly CONTET-AUDONNEAU - Madame Marie MACHOUART

$46^{\text {ème }}$ Section : SANTÉ PUBLIQUE, ENVIRONNEMENT ET SOCIÉTÉ

$1^{\text {ère }}$ sous-section : (Epidémiologie, économie de la santé et prévention)

Docteur Alexis HAUTEMANIĖRE - Docteur Frédérique CLAUDOT - Docteur Cédric BAUMANN $2^{\mathrm{e} m e}$ sous-section (Médecine et Santé au Travail) Docteur Isabelle THAON

$3^{\text {ème }}$ sous-section (Médecine légale et droit de la santé)

Docteur Laurent MARTRILLE

$4^{\text {ère }}$ sous-section : (Biostatistiques, informatique médicale et technologies de communication Docteur Nicolas JAY 
$47^{\text {ème }}$ Section : CANCÉROLOGIE, GÉNÉTIQUE, HÉMATOLOGIE, IMMUNOLOGIE

$2^{\text {ème }}$ sous-section : (Cancérologie ; radiothérapie : cancérologie (type mixte : biologique)

Docteur Lina BOLOTINE

$3^{\text {ème }}$ sous-section : (Immunologie)

Docteur Marcelo DE CARVALHO BITTENCOURT

$4^{\text {ème }}$ sous-section : (Génétique)

Docteur Christophe PHILIPPE - Docteur Céline BONNET

\title{
$48^{\text {ème }}$ Section : ANESTHÉSIOLOGIE, RÉANIMATION, MÉDECINE D'URGENCE, PHARMACOLOGIE ET THÉRAPEUTIQUE \\ $3^{\text {ème }}$ sous-section : (Pharmacologie fondamentale ; pharmacologie clinique)
}

Docteur Françoise LAPICQUE - Docteur Marie-José ROYER-MORROT - Docteur Nicolas GAMBIER

\author{
$50^{\text {ème }}$ Section : RHUMATOLOGIE \\ $1^{\text {ère }}$ sous-section : (Rhumatologie) \\ Docteur Anne-Christine RAT \\ $3^{\text {ème }}$ sous-section : (Dermato-vénéréologie) \\ Docteur Anne-Claire BURSZTEJN
}

$53^{\text {ème }}$ Section : MÉDECINE INTERNE, GÉRIATRIE et CHIRURGIE GÉNÉRALE

$1^{\text {ère }}$ sous-section : (Médecine interne ; gériatrie et biologie du vieillissement ; médecine générale ; addictologie) Docteur Laure JOLY

$54^{\text {ème }}$ Section : DÉVELOPPEMENT ET PATHOLOGIE DE L'ENFANT, GYNÉCOLOGIE-OBSTÉTRIQUE, ENDOCRINOLOGIE ET REPRODUCTION $3^{\text {ème }}$ sous-section :

Docteur Olivier MOREL

$5^{\text {ème }}$ sous-section : (Biologie et médecine du développement et de la reproduction ; gynécologie médicale) Docteur Jean-Louis CORDONNIER

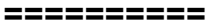

MAITTRE DE CONFÉRENCE DES UNIVERSITÉS DE MÉDECINE GÉNÉRALE Docteur Elisabeth STEYER

ヘニニニニーニニニニ

\section{MAÎTRES DE CONFÉRENCES}

$5^{\text {ème }}$ section : SCIENCE ÉCONOMIE GÉNÉRALE

Monsieur Vincent LHUILLIER

$40^{\text {ème }}$ section : SCIENCES DU MÉDICAMENT

Monsieur Jean-François COLLIN

$60^{\text {ème }}$ section : MÉCANIQUE, GÉNIE MÉCANIQUE ET GÉNIE CIVILE Monsieur Alain DURAND

$61^{\text {ème }}$ section : GÉNIE INFORMATIQUE, AUTOMATIQUE ET TRAITEMENT DU SIGNAL Monsieur Jean REBSTOCK - Monsieur Walter BLONDEL

$64^{\text {ème }}$ section : BIOCHIMIE ET BIOLOGIE MOLÉCULAIRE

Mademoiselle Marie-Claire LANHERS - Monsieur Pascal REBOUL - Mr Nick RAMALANJAONA 
$65^{\text {ème }}$ section : BIOLOGIE CELLULAIRE

Mademoiselle Françoise DREYFUSS - Monsieur Jean-Louis GELLY

Madame Ketsia HESS - Monsieur Hervé MEMBRE - Monsieur Christophe NEMOS - Madame Natalia DE ISLA Madame Nathalie MERCIER

$66^{\text {ème }}$ section : PHYSIOLOGIE

Monsieur Nguyen TRAN

$67^{\text {ème }}$ section : BIOLOGIE DES POPULATIONS ET ÉCOLOGIE

Madame Nadine MUSSE

PROFESSEURS ASSOCIÉS

Médecine Générale

Professeur associé Francis RAPHAEL

MAÎTRES DE CONFÉRENCES ASSOCIÉS

Médecine Générale

Docteur Jean-Louis ADAM

Docteur Paolo DI PATRIZIO

Docteur Sophie SIEGRIST

Docteur Arnaud MASSON

ヘニニニニニニニ

\section{PROFESSEURS ÉMÉRITES}

Professeur Jean-Marie ANDRÉ - Professeur Daniel ANTHOINE - Professeur Pierre BEY Professeur Patrick BOISSEL - Professeur Michel BOULANGÉ - Professeur Jean-Pierre CRANCE

Professeur Jean-Pierre DELAGOUTTE - Professeur Jean-Marie GILGENKRANTZ

Professeur Simone GILGENKRANTZ - Professeur Michèle KESSLER - Professeur Henri LAMBERT

Professeur Denise MONERET-VAUTRIN - Professeur Pierre MONIN - Professeur Jean-Pierre NICOLAS

Professeur Luc PICARD - Professeur Michel PIERSON - Professeur Jacques POUREL

Professeur Jean-François STOLTZ - Professeur Michel STRICKER - Professeur Gilbert THIBAUT

Professeur Hubert UFFHOLTZ - Professeur Paul VERT

Professeur Colette VIDAILHET - Professeur Michel VIDAILHET

Professeur Norman SHUMWAY (1972)

Université de Stanford, Californie (U.S.A)

Professeur Paul MICHIELSEN (1979)

Université Catholique, Louvain (Belgique)

Professeur Charles A. BERRY (1982)

Centre de Médecine Préventive, Houston (U.S.A)

Professeur Pierre-Marie GALETTI (1982)

Brown University, Providence (U.S.A)

Professeur Mamish Nisbet MUNRO (1982)

Massachusetts Institute of Technology (U.S.A)

Professeur Mildred T. STAHLMAN (1982)

Vanderbilt University, Nashville (U.S.A)

\section{DOCTEURS HONORIS CAUSA}

Harry J. BUNCKE (1989)

Université de Californie, San Francisco (U.S.A)

Professeur Daniel G. BICHET (2001)

Université de Montréal (Canada)

Professeur Brian BURCHELL (2007)

Université de Dundee (Royaume Uni)

Professeur Théodore H. SCHIEBLER (1989)

Institut d'Anatomie de Würtzburg (R.F.A)

Professeur Maria DELIVORIA-PAPADOPOULOS

(1996)

Université de Pennsylvanie (U.S.A)

Professeur Mashaki KASHIWARA (1996)

Research Institute for Mathematical Sciences de Kyoto (JAPON)

\author{
Professeur Ralph GRÄSBECK (1996) \\ Université d'Helsinki (FINLANDE) \\ Professeur James STEICHEN (1997) \\ Université d'Indianapolis (U.S.A) \\ Professeur Duong Quang TRUNG (1997) \\ Centre Universitaire de Formation et de \\ Perfectionnement des Professionnels de Santé d'Hô \\ Chi Minh-Ville (VIEETNAM) \\ Professeur Marc LEVENSTON (2005) \\ Institute of Technology, Atlanta (USA)
}


A notre Président et Maître de thèse,

Madame le Professeur Angioi-Duprez,

Professeur en Ophtalmologie,

Vous nous avez fait l'honneur de nous confier ce travail. Nous vous remercions pour la richesse de vos enseignements et la qualité de votre encadrement clinique. Travailler avec vous a toujours été un plaisir et nous sommes heureux de vous compter parmi nos Maîtres de stage. Nous vous remercions de la confiance et du soutien que vous nous apportez. Veuillez croire en notre gratitude et notre profond respect. 
A Notre Juge,

Monsieur le Professeur George,

Professeur en Ophtalmologie,

Vous nous avez fait l'honneur d'accepter de juger notre thèse et nous vous en sommes reconnaissants.

Vos connaissances et vos qualités de chirurgien sont pour nous un exemple. Vous nous apprenez avec rigueur et humilité notre travail et nous vous en remercions.

Veuillez trouver ici l'expression de notre reconnaissance et de notre profond respect. 
A notre Juge,

Monsieur le Professeur Berrod,

Professeur en Ophtalmologie,

Nous vous remercions de participer au jugement de notre travail.

Vous nous avez fait apprécier la chirurgie vitréorétinienne et nous vous en remercions. Nous vous sommes reconnaissants du savoir que vous nous avez transmis.

Que ce travail soit l'occasion pour nous de vous témoigner notre profond respect. 
A notre Juge,

Monsieur le Professeur Peyrin-Biroulet,

Professeur en Gastro-Entérologie,

Vous nous avez fait confiance pour la réalisation de ce travail et nous vous en sommes reconnaissants. Nous vous remercions infiniment pour votre présence et votre soutien tout au long de l'élaboration de cet ouvrage. Votre investissement médical et universitaire est pour nous un exemple.

Soyez assuré de notre reconnaissance et de notre plus profond respect. 
Marie-Christine Bazard: pour tout ce que tu m'as transmis au cours de ces années. Merci de ta confiance et de tes conseils. A toutes nos discussions devant les bacs de la salle 5 de Central...

Docteur Maalouf: votre rigueur et vos qualités chirurgicales sont pour moi un exemple. Je vous remercie de votre confiance.

Docteur Hubert : tes connaissances et ta disponibilité m'ont permis de progresser. Merci pour tes conseils. J'ai eu raison de t'écouter...!

Docteur Lesure : merci pour votre gentillesse et votre soutien. Paulette ne l'oubliera pas...!

Docteur Salaun : vous m'avez accueilli dans votre service pour mon tout premier stage d'ophtalmologie. Votre gentillesse et votre compréhension m'ont beaucoup touchées. Je vous remercie.

Malicia Casillas, Rym Ouled Moussa (ou plutôt arym le brancardier !), Alexandre Robin, mes CCA : vous m'avez été d'une aide précieuse pendant ces années. Je ne vous serai jamais assez reconnaissante !

A mes maîtres de stage : Professeur Sockeel, Pr Auque, Pr Marchal, Pr Civit, Dr Ract Madoux, Cédric Grasswill, Dr Huttin.

A mes co-internes et amis :

JB : pour ta générosité, ton intégrité, ton humour. Nos chemins se sont suivis depuis la P1. Sans toi, je ne serai pas là. Du fond du cœur, merci! ps : et aussi parce que grâce à toi, je ne redoute plus que l'on écorche mon nom lors de l'appel!

François : à nos révisions du dimanche $\mathrm{AM}$ et dans le métro. Merci pour ton soutien, même si «c'était vraiment horrible ! ».

Anne : à notre amitié depuis le début de notre internat. Merci infiniment pour ton soutien pendant ces 5 années, notamment dans les moments de stress la veille de l'EBO!

Alissa : pour ta bonne humeur, ta générosité et surtout ton humour! Pour le RDV raté de Jamel D, les converses bicolores et j'en passe !

Jérôme : au Zidane de la béquille ! Merci pour tous tes conseils et ton aide.

Benjamine : toujours là pour me soutenir à tout moment. Pour notre complicité et amitié. Ca n'a pas de prix. Merci...

Fanny : à ma tenue de cheval, au labrador, au Saint Seb, à la slip lamp et à tous nos fous rires. Travailler avec toi est un réel plaisir. Reviens vite de l'autre côté du champ !

Audrey: à la plus déjantée de mes co-internes. Toujours d'attaque dans toutes situations. Une belle rencontre ! à très vite, pour pleins d'autres questions...!

Soydan : mon chauffeur de Mustang. Pour ta gentillesse et ton oreille attentive. 
Andy : le pick up artist ! aux strab ensemble à l'hôpital d'enfant, toujours différents !

Constance, Shanour : jamais en stage ensemble, mais tellement contente de vous avoir comme co-internes. Merci pour votre bonne humeur, malgré votre recrutement pas toujours évident...!

Oualid : au chef !! Parce que la correction optique totale te fait mieux dormir la nuit !

Till : pour ta gentillesse et aussi pour ton « clignotant !»...

Aux secrétaires Delphine, Aurore, Cindy, Sophie, Christine, Christelle, Isabelle(s), Anne, Andrée, M. Christine. Merci de garder le sourire malgré tout ce que l'on vous demande.

A toutes les infirmières et AS : Francine, Sandrine, Nadine, Cécile, Catherine, Maryse, Elizabeth, Jocelyne, Nicole, Antoine, Karine, Ghislaine, Murielle, Fabienne, Odile, Régine... et d'hospit: Jenifer, Manue, Véronique, Virginie, Anne-Marie, Isabelle, Mireille(s)... et à toutes celles que je n'ai pas cité.

Aux équipes d'orthoptie sur qui l'on peut toujours compter: merci à Aurélia, Elizabeth, Camille, Agnès, Coline, Christelle, Chantal.

Aux internes de gastro-entérologie, qui m'ont aidé pour ce travail.

Anthony Buisson : que j'ai vouvoyé tout le long de la rédaction de cette thèse ! Merci pour ton aide.

Franck Hansmannel: pour toutes ces heures passées devant les tableaux excel. Merci! 
Cécile : bientôt 26 ans... le chemin a été long depuis la maternelle, mais nous ne nous sommes jamais quittées, même rue R. Poincaré ! Tu me fais l'honneur d'être ton témoin pour ce jour si unique et j'en suis vraiment touchée. Pour toutes ces années à venir ensemble...

Marie-Adeline : sans toi sur les bancs de la fac, je n'aurais jamais réussi cette P1. Alors pour ton écoute, ton épaule toujours à disposition pour les coups de blues, Merci !... Et rendez-vous le $1^{\text {er }}$ septembre, pour cela aussi, encore merci.

Jean-Christophe : toujours là à mes côtés. Nos parcours différents n'auront pas eu raison de notre amitié.

Maud : pour tous nos fous rires, nos coups de stress dans la véranda de Laneuneu, pour notre colloc avec pouxtra et j'en passe. Les mots sont inutiles je pense... tu sais déjà tout...

Hélène : une amie qui le vaut bien! Tellement de souvenirs en si peu de temps ! Qui aurait pu le croire aux choix à Paris ! Pour ton attention, ta disponibilité, ton humour hors pair et notre complicité...

Fanélie : pour te prouver qu'une amitié peut durer toute la vie. Pour ta sensibilité, ton grand coeur. Et parce que l'ours blanc...

Aux chouchous Julie et Fred: Pour votre soutien inégalable, votre joie de vivre. Merci.

Elodie G : Pour toutes ces longues heures à la BM. Ils n'auraient pas la même saveur sans toi. Merci de m'avoir permis de me reposer sur ton épaule.

Pour tous mes amis rencontrés à Nancy :

Thomas S (notre triangulaire !), Claire, Marie et Hub, Pilou (mon Lillois préféré : Nancy n'est plus pareil sans toi), Pierre-Etienne et Béa (sans oublier Gisèle !), Nico et Jess, Shagha, Nico, Vincent, Mathieu, Alex, Marie Lorraine... A Camille, Charline, Josépha, Manue, Emeline et toutes les autres : vive les mercredis soirs !

Et à tous ceux que j'ai croisé sur ma route, qui m'ont aidé à avancer, à construire mon avenir : merci 
A ma famille :

A Maman et Papa : vous avez toujours cru en moi et je n'ai qu'une chose à vous dire : MERCI. Maman : tu es notre pilier, notre fédérateur. Merci de nous avoir inculqué toutes ces valeurs qui te sont chères. Papa : je sais qu'aujourd'hui j'ai accompli un de tes rêves. Tu m'as constamment soutenue même si je ne le comprenais pas toujours à temps. Je suis fière d'être votre fille.

A ma sœur Céline : parce que toi seule sais combien je redoute de me retrouver devant une page blanche... et parce que tu es toujours là, dans tous mes moments de doutes, de stress (surtout !) et de bonheur. Merci !

A ma sœur Lorraine: pour tous nos crêpages de chignon, mais surtout pour nos réconciliations. A une sœur et une maman hors pair.

A Xavier : qui me supporte chez lui pendant toutes mes sessions de DU. Tu es dans la famille depuis 15 ans, tu es pour moi comme une frère. Merci d'être toujours à l'écoute.

A Joseph : je n'arriverai jamais à faire des discours aussi beaux que les tiens ! Alors merci d'être venu agrandir notre famille.

A mes grands-parents :

A Manzanne : on ne peut espérer meilleure grand-mère. Ton amour, ton écoute, et ta gentillesse nous ont fait avancer sereinement dans la vie. Merci. Et aussi à papy : pour m'avoir montré à quel point on peut être passionné par son travail.

A Mamie: Tu m'as toujours encouragée dans cette voie. Pour toutes nos vacances passées dans cette région qui est si chère à ton cœur. Merci.

A mes neveux et nièce : Arthur, Victor, Léa : de vraies terreurs...!

A mes «presque » beaux-parents, Françoise et Marc. Merci pour votre gentillesse et votre soutien. A Olivier et Guillaume.

Et à tous ceux de Remiremont :

A Jean-Richard et Yannick : j'aurai pu vous citer dans ma famille. Merci de m'avoir accueilli dans votre famille depuis plus de 25 ans.

A Gilbert et Marie-Françoise : pour tous nos cours de maths dans le jardin.

A Reza et Gilla Ebadi 
Et à Thibaut: Pour ta patience, ton aide, ta compréhension, tes encouragements pendant mes moments de doutes. Pour ta bonne humeur, ton humour, ton optimisme de chaque jour. Pour tout ce que l'on partage et pour la suite. 


\section{SERMENT}

"Au moment d'être admise à exercer la médecine, je promets et je jure d'être fidèle aux lois de l'honneur et de la probité. Mon premier souci sera de rétablir, de préserver ou de promouvoir la santé dans tous ses éléments, physiques et mentaux, individuels et sociaux.

Je respecterai toutes les personnes, leur autonomie et leur volonté, sans aucune discrimination selon leur état ou leurs convictions. J'interviendrai pour les protéger si elles sont affaiblies, vulnérables ou menacées dans leur intégrité ou leur dignité. Même sous la contrainte, je ne ferai pas usage de mes connaissances contre les lois de l'humanité. J'informerai les patients des décisions envisagées, de leurs raisons et de leurs conséquences. Je ne tromperai jamais leur confiance et n'exploiterai pas le pouvoir hérité des circonstances pour forcer les consciences. Je donnerai mes soins à l'indigent et à quiconque me les demandera. Je ne me laisserai pas influencer par la soif du gain ou la recherche de la gloire.

Admise dans l'intimité des personnes, je tairai les secrets qui me sont confiés. Reçue à l'intérieur des maisons, je respecterai les secrets des foyers et ma conduite ne servira pas à corrompre les moeurs. Je ferai tout pour soulager les souffrances. Je ne prolongerai pas abusivement les agonies. Je ne provoquerai jamais la mort délibérément.

Je préserverai l'indépendance nécessaire à l'accomplissement de ma mission. Je n'entreprendrai rien qui dépasse mes compétences. Je les entretiendrai et les perfectionnerai pour assurer au mieux les services qui me seront demandés. J'apporterai mon aide à mes confrères ainsi qu'à leurs familles dans l'adversité.

Que les hommes et mes confrères m'accordent leur estime si je suis fidèle à mes promesses ; que je sois déshonorée et méprisée si j'y manque". 


\section{$\underline{\text { Table des matières }}$}

Avant propos

p.12

Abstract

p.18

Introduction

p. 20

Material and methods

p. 22

Study population

p. 22

Ophthalmologic examination protocol

p.23

Statistical analysis

p. 24

Results

p. 25

Baseline characteristics of the patients

p. 25

Frequency and type of ocular symptoms

p. 25

Ocular lesions detected by ophthalmologic examination

p.26

Discussion

p. 27

Conclusion

p. 30

References

p. 31

Figure legends

p.36 


\section{AVANT PROPOS}

A la demande du Doyen de la faculté de Médecine de Nancy, les Thèses d'exercice doivent être écrites sous forme d'article. Je présente donc mon travail, intitulé : « Les symptômes oculaires sont-ils prédictifs d'une inflammation ophtalmologique au cours des maladies inflammatoires chroniques intestinales? Etude prospective chez 306 patients » sous cette forme. Cet article a été rédigé en anglais et proposé dans la revue « Digestive and Liver Disease ».

Néanmoins, en guise d'introduction, je propose ici un court résumé de mon travail en français.

Les buts de notre étude étaient d'étudier le caractère prédictif des manifestations oculaires dans les maladies inflammatoires chroniques intestinales (MICI) et d'évaluer l'impact des traitements de la maladie sur les manifestations ophtalmologiques chez les patients d'une large cohorte prospective.

Les maladies inflammatoires chroniques intestinales (MICI), comprenant la maladie de Crohn $(\mathrm{MC})$ et la rectocolite hémorragique $(\mathrm{RCH})$ sont des atteintes inflammatoires chroniques d'origine indéterminée. Des manifestations extraintestinales, en particulier articulaires, dermatologiques, hépatiques ou oculaires sont classiquement décrites chez les patients atteints de MICI. L'incidence des complications oculaires est estimée entre $3,5 \%$ et $12 \%$ selon les études. L'évaluation des manifestations oculaires n'est pas systématique en pratique clinique dans cette population. Cependant, détecter une inflammation oculaire comme une uvéite par exemple est très importante du fait des complications potentielles. 
Le caractère prédictif des symptômes oculaires pour l'apparition ou la présence d'une inflammation oculaire spécifique dans cette population n'a pas encore été étudié.

Nous avons réalisé une étude commune dans les services de Gastro-entérologie et d'Ophtalmologie du CHU de NANCY. Tous les patients vus de manière consécutive en consultation ou en hospitalisation dans le service de Gastro-entérologie entre avril 2009 et juillet 2011 ont répondu à un questionnaire élaboré conjointement entre les deux services. Le questionnaire incluait les items suivants : irritation oculaire, rougeur oculaire, douleur oculaire, sécrétion sur les cils, flou visuel, diplopie, myodésopsies, métamorphopsies, baisse d'acuité visuelle (BAV) progressive, BAV brutale et autre doléance. Si le patient avait au moins un symptôme oculaire, un examen ophtalmologique était systématiquement proposé au patient avec réalisation d'un examen à la lampe à fente, d'un test Tear Break-Up Time, d'un test de Schirmer et d'un fond d'œil. Afin d'évaluer les liens entre les signes ophtalmologiques et les traitements spécifiques des MICI, nous avons relevé tous les traitements systémiques liés à ces maladies tels que les anti-TNF, les immunomodulateurs, les corticoïdes et l'acide 5-amino salycilique (5-ASA).

Trois cent six patients ont participé à cette étude : 169 étaient des femmes $(55,2 \%)$, 228 étaient atteints d'une maladie de crohn (74,5\%), 77 d'une rectocolite hémorragique $(25,2 \%)$ et 1 d'une colite microscopique $(0,3 \%)$. L'âge moyen de diagnostic de la maladie était de 28,7 ans (écart type $\pm 13,2$ ). L'âge moyen à l'inclusion était de 39 ans (écart type $\pm 13,9$ ). La durée moyenne d'évolution de la maladie était de 70,4 mois. Sur les 306 patients, 112 étaient traités par infliximab $(36,6 \%), 43$ par 5-ASA oral (14\%), 41 par adalinumab (13,4\%), 25 par azathioprine (8,2\%), 19 par corticoïdes $(6,2 \%), 16$ par méthotrexate $(5,2 \%), 9$ par budésonide 
(2,9\%), 1 par ciclosporine $(0,3 \%), 2$ par certolizumab pégol $(0,6 \%)$ et 2 par mycophénolate mofétil $(0,6 \%)$. Quatre-vingt dix huit patients $(32 \%)$ ont rapporté au moins un symptôme oculaire : irritation oculaire $(56,8 \%)$, rougeur oculaire $(40,5 \%)$, flou visuel $(37,8 \%)$, douleur oculaire $(31,3 \%)$, BAV progressive $(34,4 \%)$, myodésopsies $(23,3 \%)$, sécrétions sur les cils $(12,2 \%)$, œil sec $(9,5 \%)$, larmoiement $(6,8 \%)$, diplopie $(5,4 \%)$, métamorphopsies $(4 \%)$ et BAV brutale $(4 \%)$.

L'examen ophtalmologique a été réalisé chez 74 patients. Les 24 autres patients ne sont pas venus au rendez-vous proposé. Les atteintes suivantes ont été mises en évidence: une sécheresse oculaire dans $41,9 \%$ des cas, une kératite ponctuée superficielle dans $23 \%$ des cas, une blépharite dans $14,9 \%$ des cas et une conjonctivite dans $9,5 \%$ des cas. Une sclérite était retrouvée chez un patient. Aucune uvéite n'a été diagnostiquée. En analyse univariée, aucun traitement n'était associé à la sécheresse oculaire.

Cette étude est l'une des plus larges séries prospectives consacrées à l'ophtalmologie dans une population de patients atteints de MICI. Dans notre série, les symptômes oculaires sont fréquents chez les patients porteurs de MICI, mais ne sont que très rarement associés à des atteintes inflammatoires oculaires spécifiques.

Une seule étude a recensé la fréquence et le type de symptômes oculaires rapportés par les patients atteints de MICI. Elle n'a malheureusement pas évalué leur association avec une inflammation oculaire.

Notre étude rapporte une prévalence élevée de sécheresse oculaire $(41,9 \%)$ avec des résultats similaires à ceux des données de la littérature. Une inflammation de bas grade liée à la présence d'auto-anticorps dans la glande lacrymale ou une hypovitaminose A lié à une malabsorption pourrait expliquer ces manifestations 
oculaires. Nous n'avons cependant pas relevé les autres traitements pouvant induire cette iatrogénicité (tels les antidépresseurs tricycliques).

En conclusion, notre étude a permis de montrer que les manifestations oculaires sont fréquentes chez les patients atteints de MICI, mais qu'elles sont rarement associées à une inflammation oculaire. Un interrogatoire systématique à la recherche de symptômes oculaires ne semble pas être recommandé dans la pratique clinique. 


\section{OCULAR SYMPTOMS ARE NOT PREDICTIVE OF OPHTHALMOLOGIC INFLAMMATION IN INFLAMMATORY BOWEL DISEASE}

Running head: Ocular symptoms and inflammation in IBD

Véronique Cloché ${ }^{1} \mathrm{MD}$; Anthony Buisson ${ }^{2,3,4}$, MD; Fanny Tréchot ${ }^{1}$ MD; Benjamine

Batta ${ }^{1}$ MD; Andy Locatelli ${ }^{1}$ MD; Constance Favel ${ }^{1}$ MD; Shanour Premy ${ }^{1}$ MD;

Benjamin Collet-Fenestrier ${ }^{2}$, MD; Estelle Freling ${ }^{2}$, MD; Anthony Lopez ${ }^{2}$, MD; Marie Pauline Massoure ${ }^{2}, \mathrm{MD}$; Anne-Laure Humbert ${ }^{2}$, MD; Franck Hansmannel ${ }^{2} \mathrm{PhD}$; JeanLouis Guéant, $\mathrm{MD}, \mathrm{PhD}^{2}$; Marc-André Bigard ${ }^{2} \mathrm{MD}$, Laurent Peyrin-Biroulet ${ }^{2} \mathrm{MD}$, $\mathrm{PhD}$ *; Karine Angioi ${ }^{1} \mathrm{MD}, \mathrm{PhD}$ *.

${ }^{1}$ Department of Ophthalmology, Nancy University Hospital, Vandoeuvre-lès-Nancy;

${ }^{2}$ Inserm U954 and Department of Hepato-Gastroenterology, University Hospital of Nancy, Université Henri Poincaré 1, Vandoeuvre-lès-Nancy;

${ }^{3}$ Microbes, intestine, inflammation and susceptibility of the host, UMR Inserm/Université d'Auvergne U1071, USC-INRA 2018, Clermont Université, Clermont-Ferrand;

${ }^{4}$ Department of Hepato-Gastroenterology, University Hospital Estaing of ClermontFerrand, Université d'Auvergne, Clermont-Ferrand, France.

*These authors share senior authorship. 
Corresponding author:

Prof. Karine Angioi, MD, PhD

Department of Ophthalmology, University Hospital of Nancy-Brabois, Université Henri Poincaré 1, Allée de Morvan, 54511 Vandoeuvre-lès-Nancy, France

E-mail: k.angioi-duprez@,chu-nancy.fr

AND

Prof. Laurent Peyrin-Biroulet, MD, PhD

Inserm U954 and Department of Hepato-Gastroenterology, University Hospital of Nancy-Brabois, Université Henri Poincaré 1, Allée du Morvan, 54511 Vandoeuvrelès-Nancy, France

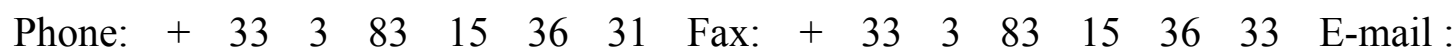
peyrinbiroulet@gmail.com

Key words: Inflammatory bowel disease, ocular symptoms, ocular inflammation 


\begin{abstract}
Background: Ocular manifestations are frequent in patients with inflammatory bowel disease (IBD). Whether ocular symptoms predict the presence of inflammatory ocular diseases in IBD is unknown.
\end{abstract}

Aim: To evaluate the value of ocular symptoms in predicting ophthalmologic inflammation in patients with IBD.

Methods: All consecutive IBD patients seen in the Department of Gastroenterology (Nancy, University Hospital, France) between April 2009 and July 2011 were interviewed for this cross-sectional study using a pre-established questionnaire. This questionnaire was developed by the Departments of Gastroenterology and Ophthalmology. If the patient had at least one ocular symptom, he systematically underwent an ophthalmologic examination (visual acuity, Tear Break-Up Time test, Schirmer test, slit-lamp exam with fundus examination).

Results: This cross-sectional survey was completed by 306 patients: 169 were women (55.2\%), 228 had Crohn's disease (74.5\%), 77 ulcerative colitis (25.2\%) and 1 microscopic colitis $(0.3 \%)$. Ninety-eight patients $(32 \%)$ reported at least one ocular symptom: ocular irritation (56.8\%), red eye (40.5\%), blurred vision (37.8\%), ocular pain (31.1\%), progressive visual loss (34.4\%), myodesopsia (23.3\%), eyelid secretion (12.2\%), dry eye (9.5\%), watering (6.8\%), diplopia (5.4\%), metamorphopsia (4\%), and sudden visual loss (4\%). Following ophthalmologic examination $(n=74), 41.9 \%$ patients had evidence of dry eye $(n=31), 14.9 \%$ blepharitis $(n=11)$ and $1.4 \%$ scleritis $(n=1)$. No uveitis was reported. No factors were associated with dry eye. 
Conclusion: Ocular symptoms are frequent in IBD patients, but are non-specific and rarely associated with ocular inflammation. Systematic ocular symptoms assessment is of poor value for diagnosing ocular inflammation in clinical practice in IBD patients. 


\section{INTRODUCTION}

Inflammatory bowel disease (IBD) encompassing Crohn's disease (CD) and ulcerative colitis (UC) are chronic inflammatory disorders of unknown aetiology (1) Extraintestinal manifestations, including joint, skin, liver, ocular involvement are common in IBD (2-4). The incidence of ocular complications ranged from $3.5 \%$ to $12 \%(5-11)$.

In a referral center study, ophthalmologic manifestations were reported by up to $43 \%$ of IBD patients (5). Various ocular lesions have been observed among IBD patients such as episcleritis, scleritis, keratopathy, anterior uveitis, papillitis, neurobulbar neuritis, central serous chorioretinopathy, choroiditis, vasculitis, orbital cellulitis, myositis, orbital pseudotumor (6-21). Ophthalmologic complications can be categorized as primary, secondary and coincidental (7). Primary ocular complications occur during flares of the disease and are correlated to disease activity. IBD therapies are often effective in treating these manifestations, including keratophathy, anterior stromal and limbal infiltrates, episcleritis, scleritis, uveitis, macular edema, central serous retinopathy, and proptosis. Secondary ocular manifestations result from primary, systemic or ocular complications (e.g cataract, scleromalacia, exsudative retinal detachment, optic disk edema, decreased tear formation, night blindness, candida endophtalmitis). Coincidental complications are frequent in the general population and unrelated to IBD (e.g conjunctivitis, recurrent corneal erosion, corneal ulcer, glaucoma, generalized retinal arterial narrowing, subcunjonctival hemorrhage) (7).

Assessment of ocular manifestations is not systematically done in clinical practice in IBD. However, the detection of ocular inflammation (uveitis etc.) is very important as complications such as posterior synechia, cataract, hypertonia, ischemic vasculitis, 
macular oedema, and corneal perforation may occur in these patients (11). In all available studies on ophthalmologic manifestations in IBD, only systematic ocular examination was performed irrespective of the symptoms $(4,5,7,10,11,22)$. However, systematic ophthalmologic examination cannot be recommended in clinical practice. The only study which assessed both symptoms and ocular inflammation did not report whether symptoms were correlated to ocular inflammation (11). Hence, whether ocular symptoms predict the presence of inflammatory ocular diseases and should be systematically screened in clinical practice in inflammatory bowel disease (IBD) has yet to be determined. The aims of this study were therefore to assess the predictive value of ocular symptoms for predicting ocular inflammation and the impact of medications on ophthalmologic manifestations in a large prospective cohort of IBD patients. By definition, such study design does not require a control group as our aim was not to evaluate the prevalence of ocular lesions in IBD. 


\section{MATERIALS AND METHODS}

\section{Study population}

In the department of Gastroenterology of Nancy University Hospital, all IBD patients are systematically and routinely interviewed for extra-intestinal manifestations. All consecutive inpatients and outpatients IBD patients seen at the IBD Unit of Nancy University Hospital between April 2009 and July 2011 were included in this prospective observational study. The data were retrieved from the Nancy IBD cohort using patients' electronic charts. Information about the Nancy IBD cohort is reported to the Commission Nationale de l'Informatique et des Libertés (no.1404720), which supervises the implementation of the act regarding data processing, data files and individual liberties that came into effect on 6 January 1978, and was amended on 6 August 2004, to protect the personal data of individuals (23-25).

A pre-established questionnaire was developed by the Departments of Gastroenterology and Ophthalmology and encompassed the following items: ocular irritation, redness, ocular pain, eyelid secretion, blurred vision, diplopia, myodesopsia, metamorphopsia, progressive visual loss, sudden visual loss and others (verbatim analysis). All patients enrolled in this study were interviewed using this questionnaire. If the patient had at least one ocular symptom, an ophthalmologic examination was systematically performed as it is routinely done in our department.

All patients had an established diagnosis of IBD for at least 3 months according to Lennard Jones criteria (26). CD phenotype was classified using the Montreal Classification, including age of diagnosis (A), anatomical distribution (L), disease behaviour (B) and perianal disease (p). 
UC extent according to the Montreal classification was defined in 3 groups (E1: involvement limited to the rectum, E2: involvement limited to a proportion of the colorectum distal to the splenic flexure, E3: involvement extends proximal to the splenic flexure).

All concomitant IBD-related medications such as anti-TNF therapy, immunomodulators, corticosteroids, and 5-ASA were prospectively recorded.

\section{Ophthalmologic examination protocol}

One experienced ophthalmologist (K.A.) examined all patients who had at least one ocular symptom. Each patient underwent full ophthalmologic examination including visual acuity with the Monoyer and Parinaud tests, slit-lamp examination of the eyelid, conjonctiva, cornea, pupils, iris, and fundus examination. The intraocular pression was measured by non-contact air puff. The lacrymal film was studied using the Schirmer Test and the Fluorescein Break-Up Time Test (BUT).

For the Schirmer test, a $35 \mathrm{~mm} \times 5 \mathrm{~mm}$ size filter paper strip was used without corneal anaesthesia. The strip was placed in the middle third and the lateral third of the inferior eyelid. We counted the amount of tears produced during 5 minutes. Patient with dry eye was wetting values of less than $5 \mathrm{~mm}$ in 5 minutes.

For the BUT: a drop of fluorescein was applied in the lower eyelid fornix. The tear film was observed under cobalt-blue filtered light and the time that elapsed between the last blink and the appearance of the first break in the tear film was recorded. The eye was considered like dry eye if the BUT was less than 10 seconds.

We considered as dry eyed patients, those who have at least one of both test positive.

If the fundus examination was doubtful, an Optical Coherence Tomography was performed. 


\section{Statistical analysis}

The data were entered into a database (Microsoft Office Excel). Data analysis was carried out with StatView 5.0.1 (SAS Institute Inc, Cary, NC). Univariate comparisons of ophthalmologic lesions and treatments were accomplished using the Chi-Square test, or the Fisher exact's test if any cell number was $<5$. $\mathrm{P}<0.05$ was considered as statistically significant. 


\section{RESULTS}

\section{Baseline characteristics of the patients}

Three hundred and six patients were enrolled in this cross-sectional study: 169 were women (55.2\%), 228 had CD (74.5\%), $77 \mathrm{UC}(25.2 \%)$ and 1 had microscopic colitis $(0.3 \%)$ (Table1).

Mean age at the IBD diagnosis was 28.7 years (range \pm 13.2 ). Mean age at inclusion was 39 years (range \pm 13.9 ). Mean disease duration was 70.4 months. IBD phenotype is given in Table 1.

Of the 306 patients, 112 were treated with infliximab (36.6\%), 43 with oral 5-ASA (14\%), 41 with adalimumab (13.4\%), 25 with azathioprine (8.2\%), 19 with corticosteroids $(6.2 \%), 16$ with methotrexate $(5.2 \%), 9$ with budesonide $(2.9 \%), 1$ with ciclosporin $(0.3 \%), 2$ with certolizumab pegol $(0.6 \%)$, and 2 with mycophenolate mofetil $(0.6 \%)$ (Table 1$)$.

\section{Frequency and type of ocular symptoms}

Ninety-eight patients (32\%) reported at least one ocular symptom: ocular irritation (56.8\%), red eye $(40.5 \%)$, blurred vision $(37.8 \%)$, ocular pain $(31.1 \%)$, progressive visual loss $(34.4 \%)$, myodesopsia $(23.3 \%)$, eyelid secretion $(12.2 \%)$, dry eye $(9.5 \%)$, watering (6.8\%), diplopia (5.4\%), metamorphopsia (4\%) and sudden visual loss (4\%) (Figure 1). 


\section{Ocular lesions detected by ophthalmologic examination}

Ophthalmologic examination was completed in 74 patients (IBD phenotype and IBD treatment are given in table 1). Following the examination, $41.9 \%$ patients had evidence of dry eye, $23 \%$ superficial punctual keratitis, $14.9 \%$ blepharitis, and $9.5 \%$ cunjunctivitis. Scleritis prevalence was $1.4 \%$ (95\% confidence interval: $-0.21,0.24)$. No uveitis was diagnosed. According to the classification proposed by Knox et al. (7), primary, secondary and coincidental complications were reported in $1.4 \%$ (scleritis), $41.9 \%$ (dry eye), and $36.5 \%$ of cases (blepharitis, cunjunctivitis, superficial punctual keratitis), respectively.

There was no significant difference in demographic features between patients with or without dry eye and those with or without blepharitis.

In univariate analysis, we did not find any relationship between dry eye and the other IBD-related medications (Table 2). IBD medications were not associated with blepharitis (Table 2).

Treatment for scleritis was local dexamethasone (eye drops for 10 days), and for blepharitis eyelid cares and artificial tears. 


\section{DISCUSSION}

This is the largest study investigating the frequency and type of ocular symptoms among IBD patients. It is also the first study assessing the association of ocular symptoms with ophthalmologic inflammatory diseases in IBD.

Only one study reported frequency and type of ocular symptoms in the IBD population (27). This prospective cohort included only 112 subjects (48 patients with CD, 40 with UC and 24 controls). Ophthalmologic exam was completed in all patients. In the IBD group, eye symptoms occurred in $44 \%$ of the patients, compared to $13 \%$ in the control group. In addition $40 \%$ of $\mathrm{CD}$ patients and $50 \%$ of UC patients experienced eye symptoms. Three types of symptoms were described by IBD patients, namely itching (30\%), burning (13\%), and visual disturbance (2\%). Only $2(12 \%)$ patients of the control population had eye symptoms, and they both reported itching. Ophthalmologic exam found $43 \%$ of dry eye, $1 \%$ of conjunctivitis, $3 \%$ of blepharitis, $1 \%$ of episcleritis, $2 \%$ of uveitis, and $2 \%$ of cataract, but no scleritis, vasculitis or neuritis. Dry eye was significantly more common in IBD patients than controls (OR $5.2,95 \%$ CI 1.4-19, $p=0.008)(27)$.

From our study, $32 \%$ of the patients reported ocular symptoms. Compared to data retrieved from literature, ocular irritation and blurred vision were more frequent in our study (56.8\% vs. $13 \%$, and $37.8 \%$ vs. $2 \%$, respectively) (27). No itching was reported by the patients. Prevalence of dry eye was similar to that reported by Cury et al. (41.9\% vs. $43 \%)$ (27). Whether eye symptoms were associated with specific ocular lesions was not investigated in this study (27).

A significant proportion of IBD patients are complaining of ocular symptoms. However, a systematic ophthalmologic examination cannot be recommended in 
clinical practice. It is the reason why we decided to evaluate for the first time the predictive value of ocular symptoms for diagnosing specific ocular inflammation in IBD. While almost one third of patients reported ocular symptoms, ocular lesion (scleritis) was identified in only one patient. We can conclude that ocular symptoms are not specific and not associated with ocular inflammation.

We reported a prevalence of dry eye of $41.9 \%$ in our patients. A Greek referral center study found dry eyes in $22 \%$ of IBD patients compared to $11 \%$ of controls (5). Dry eyes were significantly more common in IBD patients than controls $(43 \%$ vs. $12 \%$, respectively) in a Brazilian center (27). This symptom is common burden in the general population; in study enrolling 654 subjects older than 40 years-old, $11 \%$ had dry eyes (28). The underlying mechanisms for this higher prevalence of dry eyes in IBD patients than in the general population remain unclear. Low-grade inflammation due to presence of auto-antibodies for lacrimal tissue (27) or hypovitaminosis A explained by malabsorption may underlie such ocular manifestation (7).

We reported a low prevalence of ocular lesions (1.4\%) in our study. Importantly, only patients having ocular symptoms underwent ophthalmologic examination according to our clinical practice. Hence, our results reflect a real-life setting. The rate of ocular lesions in IBD ranged from $4 \%$ to $30 \%$ in the literature $(5,9)$. Among the $6.3 \%$ of patients experiencing primary complications, Hopkins et al. found uveitis, corneal ulcer, episcleritis, and macular haemorrhage in $2.4 \%, 1.2 \%, 0.6 \%$, and $0.3 \%$ of cases, respectively (10). Primary ocular disease occurred in 3 out of patients in another study, with 1 episcleritis and 2 uveitis. (27). The same authors reported ocular manifestations in up to $43 \%$ of patients, including primary, secondary and coincidental complications. Among the primary complications, episcleritis was diagnosed in $2(7.7 \%)$ patients, iridocyclitis in $3(11.5 \%)$ patients, choroiditis in 1 
patient, retinal vasculitis in 1 patient, and optic neuritis in 1 patient. During a 4-year period in the University of Cape Town, South Africa, the authors examined 19 patients with Crohn's disease and associated ocular inflammation. Seven patients had uveitis, eight had episcleritis, and four had anterior scleritis. Large peripheral corneal infiltrates developed in two patients with scleritis (11).

Two explanations may underlie the low rate of ocular inflammation in our large cohort of IBD patients. First, in the era of biologics, a large part of our patients were treated with immunomodulators and/or anti-TNF agents $(36.6 \%$ with infliximab and $13.4 \%$ with adalimumab), which may decrease ocular inflammation (29-34). In line with our findings, the prevalence of subclinical anterior uveitis in adult IBD patients was low in a recent report; one hundred seventy-nine consecutive IBD patients without previous or concurrent ocular symptoms underwent ophthalmologic examination with quantitative flare measurements and were compared to a control group (35). No differences were observed between the 2 groups (35). Second, specific ocular inflammation could be asymptomatic in some patients while only patients who had ocular symptoms underwent ophthalmologic examination in our study. Interestingly, Felekis et al. diagnosed choroiditis in 1 patient, retinal vasculitis in 1 patient and optic neuritis in 1 patient among a population without history of ophthalmologic symptoms or disease (5).

Among ocular symptoms, the association of baseline characteristics with ocular lesions could be assessed only for dry eye due to a sufficient number of events $(41.9 \%$ of patients). No CD-related medications were associated with dry eye. Whether other medications, such as antidepressants, may have caused dry eye could not be investigated in our study. 
The main strengths of our study are its prospective design and the large sample size $(n=306)$. In addition, consecutive patients were enrolled in this study and all patients were included in the Nancy IBD cohort which collects systematically and accurately all the data about IBD patients (23-25). The main limitations could be the absence of systematic ophthalmologic examination and the high number of patients treated with biologics. However, the aim of our study was to assess for the first time the predictive value of ocular symptoms in diagnosing ocular inflammation in a real life setting in the era of biologics.

In conclusion, ocular manifestations are frequent in IBD patients, but are nonspecific and rarely associated with ocular inflammation. Systematic assessment of ocular symptoms appears to be of poor value in clinical practice. 


\section{REFERENCES}

1. Peyrin-Biroulet L, Desreumaux P, Sandborn WJ, et al. Crohn's disease: beyond antagonists of tumour necrosis factor. Lancet 2008;372:67-81.

2. Peyrin-Biroulet L, Loftus EV Jr, Colombel J-F, et al. Long-term complications, extraintestinal manifestations, and mortality in adult Crohn's disease in population-based cohorts. Inflamm Bowel Dis 2011;17:471-8.

3. Juillerat P, Mottet C, Pittet V, et al. Extraintestinal manifestations of Crohn's disease. Digestion 2007;76:141-8.

4. Lakatos L, Pandur T, David G, et al. Association of extraintestinal manifestations of inflammatory bowel disease in a province of western Hungary with disease phenotype: results of a 25-year follow-up study. World J Gastroenterol $2003 ; 9: 2300-7$.

5. Felekis T, Katsanos K, Kitsanou M, et al. Spectrum and frequency of ophthalmologic manifestations in patients with inflammatory bowel disease: a prospective single-center study. Inflamm Bowel Dis 2009;15:29-34.

6. Ghanchi FD, Rembacken BJ. Inflammatory bowel disease and the eye. Surv Ophthalmol 2003;48:663-76.

7. Knox DL, Schachat AP, Mustonen E. Primary, secondary and coincidental ocular complications of Crohn's disease. Ophthalmology 1984;91:163-73.

8. Rankin GB, Watts HD, Melnyk CS, Kelley ML Jr. National Cooperative Crohn's Disease Study: extraintestinal manifestations and perianal complications. Gastroenterology 1979;77:914-20. 
9. Greenstein AJ, Janowitz HD, Sachar DB. The extra-intestinal complications of Crohn's disease and ulcerative colitis: a study of 700 patients. Medicine (Baltimore) 1976;55:401-12.

10. Hopkins DJ, Horan E, Burton IL, Clamp SE, et al. Ocular disorders in a series of 332 patients with Crohn's disease. Br J Ophthalmol 1974 ;58:732-7.

11. Salmon JF, Wright JP, Murray AD. Ocular inflammation in Crohn's disease. Ophthalmology 1991;98:480-4.

12. Matsuo T, Yamaoka A. Retinal vasculitis revealed by fluorescein angiography in patients with inflammatory bowel disease. Jpn J Ophthalmol 1998;42:398-400.

13. Angioi K, Kaminsky P, Peyrin-Biroulet L. Infliximab for severe peripheral ulcerative keratopathy revealing Crohn's disease. Inflamm Bowel Dis 2011;17:866-7.

14. Diaz-Valle D, Benitez del Castillo JM, Fernandez Aceñero MJ, et al. Bilateral lid margin ulcers as the initial manifestation of Crohn disease. Am J Ophthalmol $2004 ; 138: 292-4$.

15. Tappeiner C, Dohrmann J, Spital G, et al. Multifocal posterior uveitis in Crohn's disease. Graefes Arch Clin Exp Ophthalmol 2007;245:457-9.

16. Rosenbaum JT, Kurz D. An old crone finds a new home: Crohn's disease and pars planitis. Ocul Immunol Inflamm 2002;10:157-60.

17. Chowers I, Siganos CS, Solomon A, et al. Deep stromal inflammation--a possible new ocular manifestation of Crohn's disease. Ocul Immunol Inflamm 1998;6:59-62. 
18. Pham M, Chow CC, Badawi D, et al. Use of infliximab in the treatment of peripheral ulcerative keratitis in Crohn disease. Am J Ophthalmol 2011;152:183-188.

19. Tan MH, Chen SDM, Rubinstein A, et al. Corneal perforation due to severe peripheral ulcerative keratitis in Crohn disease. Cornea 2006;25:628-30.

20. Kelly IM, Frith PA, Hyman NM, et al. Retinal periphlebitis in ulcerative colitis. Postgrad Med J 1990;66:565-7.

21. Durno CA, Ehrlich R, Taylor R, et al. Keeping an eye on Crohn's disease: orbital myositis as the presenting symptom. Can J Gastroenterol 1997;11:497-500.

22. Yilmaz S, Aydemir E, Maden A, et al. The prevalence of ocular involvement in patients with inflammatory bowel disease. Int J Colorectal Dis 2007;22:1027-30.

23. Williet N, Pillot C, Oussalah A, et al. Incidence of and impact of medications on colectomy in newly diagnosed ulcerative colitis in the era of biologics. Inflamm Bowel Dis 2011. doi: 10.1002/ibd.21932 [In press].

24. Peyrin-Biroulet L, Pillot C, Oussalah A, et al. Urinary tract infections in hospitalized inflammatory bowel disease patients: A 10-year experience. Inflamm Bowel Dis 2011. doi:

10.1002/ibd.21777 [In press].

25. Peyrin-Biroulet L, Oussalah A, Williet N, et al. Impact of azathioprine and tumour necrosis factor antagonists on the need for surgery in newly diagnosed Crohn's disease. Gut 2011;60:930-6.

26. Lennard-Jones JE. Classification of inflammatory bowel disease. Scand J Gastroenterol (Suppl. 1989);170:2-6. 
27. Cury DB, Moss AC. Ocular manifestations in a community-based cohort of patients with inflammatory bowel disease. Inflamm Bowel Dis 2010;16:1393-6.

28. Viso E, Gude F, Rodríguez-Ares MT. The association of meibomian gland dysfunction and other common ocular diseases with dry eye: a population-based study in Spain. Cornea 2011;30:1-6.

29. Colombel JF, Sandborn WJ, Rutgeerts P, et al. Adalimumab for maintenance of clinical response and remission in patients with Crohn's disease: the CHARM trial. Gastroenterology 2007;132:52-65.

30. Hanauer SB, Sandborn WJ, Rutgeerts P, et al. Human anti-tumor necrosis factor monoclonal antibody (adalimumab) in Crohn's disease: the CLASSIC-I trial. Gastroenterology 2006;130:323-33.

31. Sandborn WJ, Feagan BG, Stoinov S, et al. Certolizumab pegol for the treatment of Crohn's disease. N Engl J Med 2007;357:228-38.

32. Schreiber S, Khaliq-Kareemi M, Lawrance IC, et al. Maintenance therapy with certolizumab pegol for Crohn's disease. N Engl J Med 2007;357:239-50. 33. Targan SR, Hanauer SB, van Deventer SJ, et al. A short-term study of chimeric monoclonal antibody cA2 to tumor necrosis factor alpha for Crohn's disease. Crohn's Disease cA2 Study Group. N Eng1 J Med 1997;337:1029-35. 34. Hanauer SB, Feagan BG, Lichtenstein GR, et al. Maintenance infliximab for Crohn's disease: the ACCENT I randomised trial. Lancet 2002;359:1541-9. 
35. Verbraak FD, Schreinemachers MC, Tiller A, et al. Prevalence of subclinical anterior uveitis in adult patients with inflammatory bowel disease. $\mathrm{Br} \mathrm{J}$ Ophthalmol 2001;85:219-21. 


\section{FIGURE LEGENDS}

Figure 1: Type and frequency of ocular symptoms in IBD patients $(n=98)$.

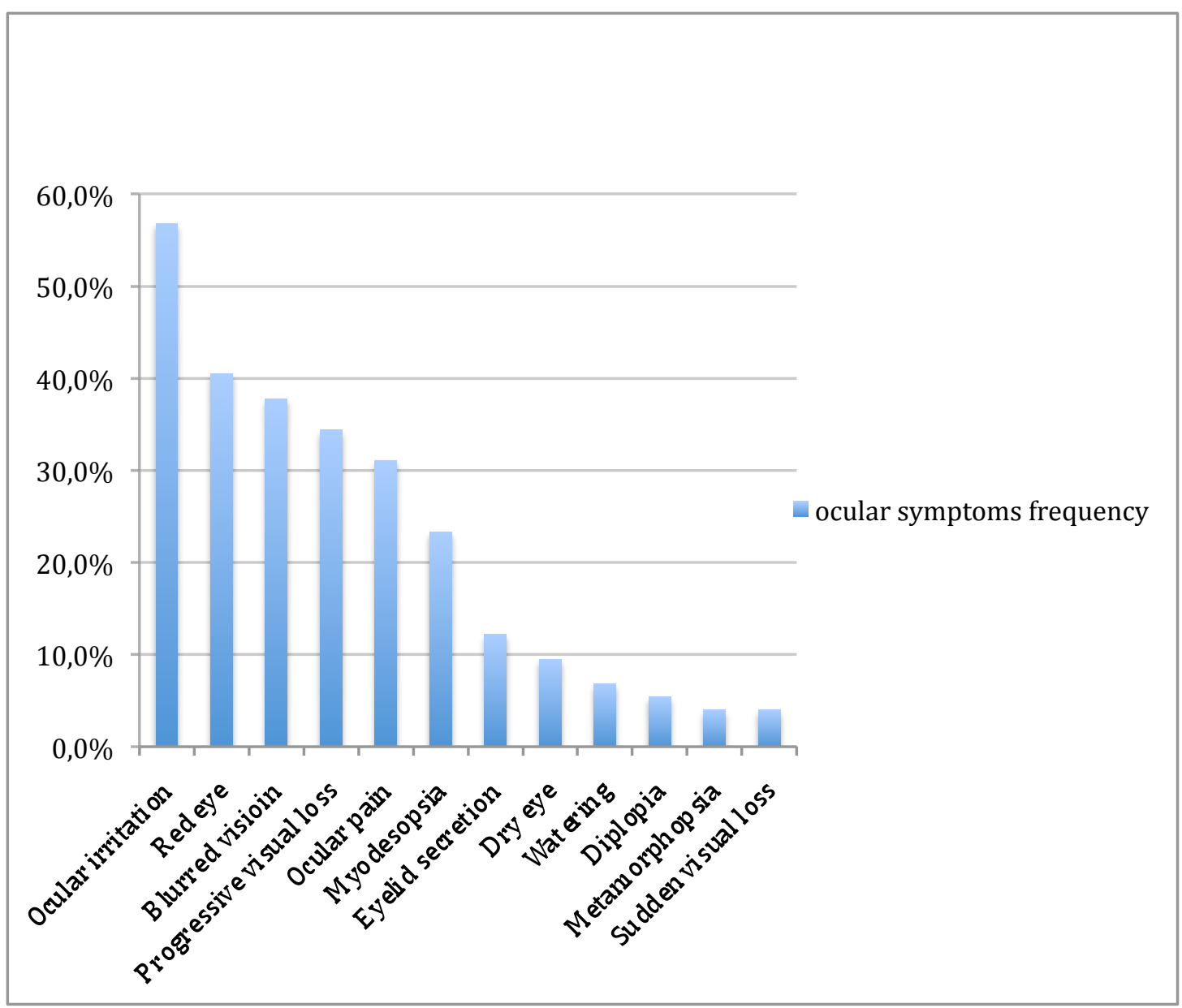


Table 1: Characteristics of the 306 patients (questionnaire) and of 74 patients (examined by ophthalmologist).

\begin{tabular}{|c|c|c|}
\hline & $\mathrm{N}(=306)$ & $\mathrm{N}(=74)$ \\
\hline Median age & $39.9( \pm 13.9)$ & $42( \pm 14.4)$ \\
\hline Female (\%) & 55.2 & $64.8 \%$ \\
\hline $\begin{array}{l}\text { Disease duration } \\
\text { (months) }\end{array}$ & $70.4( \pm 82)$ & $67.4( \pm 80.6)$ \\
\hline CD: & 228 & 55 \\
\hline Disease phenotype & & \\
\hline $\mathrm{L} 1$ & 50 & 13 \\
\hline L2 & 42 & 10 \\
\hline L3 & 134 & 32 \\
\hline L4 & 9 & 0 \\
\hline B1 & 123 & 29 \\
\hline B2 & 66 & 15 \\
\hline B3 & 39 & 11 \\
\hline $\mathrm{P}$ & 79 & 20 \\
\hline UC: & 77 & 18 \\
\hline Disease phenotype & & \\
\hline E1 & 16 & 2 \\
\hline E2 & 15 & 4 \\
\hline E3 & 46 & 12 \\
\hline $\begin{array}{l}\text { Indeterminate colitis } \\
\text { Treatments }\end{array}$ & 1 & 1 \\
\hline Infliximab & $112(36.6 \%)$ & $34(45.9 \%)$ \\
\hline 5-ASA & $43(14 \%)$ & $6(8.1 \%)$ \\
\hline Adalimumab & $41(13.4 \%)$ & $7(9.4 \%)$ \\
\hline Azathioprine & $25(8.2 \%)$ & $4(5.4 \%)$ \\
\hline Corticosteroids & $19(6.2 \%)$ & $5(6.8 \%)$ \\
\hline Methotrexate & $16(5.2 \%)$ & $9(12.2 \%)$ \\
\hline Budesonide & $9(2.9 \%)$ & $4(5.4 \%)$ \\
\hline Certolizumab pegol & $2(0.6 \%)$ & $1(1.35 \%)$ \\
\hline $\begin{array}{r}\text { Mycophenolate } \\
\text { mofetil }\end{array}$ & $2(0.6 \%)$ & 0 \\
\hline Ciclosporin & $1(0.3 \%)$ & 0 \\
\hline Symptoms & 98 & \\
\hline
\end{tabular}

IBD: inflammatory bowel disease; CD: Crohn's disease;

UC: ulcerative colitis. 
Table 2: Ocular lesions detected by ophthalmologic examiation $(n=74)$; univariate analysis.

\begin{tabular}{|cccccc|}
\hline & Scleritis & $\begin{array}{c}\text { Dry eye/No } \\
\text { dry eye }\end{array}$ & P-value & $\begin{array}{c}\text { Blepharitis/No } \\
\text { blepharitis }\end{array}$ & P-value \\
Infliximab & 1 & $15 / 11$ & 1 & $6 / 28$ & 0.50 \\
Local 5-ASA & 0 & $0 / 1$ & 0.4 & $0 / 1$ & 1 \\
Oral 5-ASA & 0 & $2 / 1$ & 1 & $0 / 5$ & 1 \\
Budesonide & 0 & $1 / 2$ & 0.56 & $0 / 4$ & 1 \\
Steroids & 0 & $2 / 1$ & 1 & $1 / 4$ & 1 \\
Ciclosporin & 0 & $0 / 0$ & & $0 / 0$ & \\
Adalimumab & 0 & $1 / 2$ & 0.56 & $0 / 7$ & 0.59 \\
Certolizumab & 0 & $0 / 1$ & 0.42 & $0 / 1$ & 1 \\
Mycophenolate & 0 & $0 / 0$ & & $0 / 0$ & \\
mofetil & & & & & \\
Azathioprine & 0 & $2 / 1$ & 1 & $1 / 3$ & 0.44 \\
Methotrexate & 0 & $2 / 4$ & 0.22 & $0 / 9$ & 0.34 \\
\hline
\end{tabular}




\section{RÉSUMÉ DE LA THÈSE}

Introduction: étudier le caractère prédictif des manifestations oculaires dans les maladies inflammatoires chroniques intestinales (MICI).

Méthode : d'avril 2009 à juillet 2011, tous les patients atteints de MICI vus de façon consécutive dans le département de Gastro-Entérologie du CHU de Nancy ont répondu à un questionnaire pré-établi réalisé conjointement par les Départements de Gastro-Entérologie et d'Ophtalmologie. Lorsqu'un patient avait au moins un symptôme, il bénéficiait d'un examen ophtalmologique systématique (acuité visuelle, test de Break-Up Time, test de Schirmer, examen à la lampe à fente avec fond d'œil). Le type de traitement était relevé.

Résultats : cette étude transversale a été réalisée chez 306 patients. Cent soixante-neuf étaient des femmes (55.2\%) et 228 étaient porteurs d'une maladie de Crohn (74.5\%), 77 d'une rectocolite hémorragique $(25.2 \%)$ et 1 d'une colite microscopique $(0.3 \%)$. Quatre-vingt huit patients (32\%) ont rapporté au moins un symptôme oculaire; l'examen ophtalmologique a permis de mettre en évidence une sécheresse oculaire (41.9\%), une blépharite $(14.9 \%)$ et une sclérite $(1.4 \%)$. Aucune uvéite n'a été rapportée. Aucun traitement n'était associé à une sécheresse oculaire.

Conclusion : les symptômes oculaires sont fréquents dans la population atteinte de MICI, mais rarement associés à une inflammation oculaire spécifique. Un interrogatoire systématique à la recherche de manifestations ophtalmologiques chez les patients atteints de MICI ne semble donc pas recommandé dans la pratique clinique.

\section{TITRE EN ANGLAIS}

Ocular symptoms are not predictive of ophthalmologic inflammation in inflammatory bowel disease

\section{THÈSE : MÉDECINE SPÉCIALISÉE - ANNÉE 2012}

MOTS CLEFS : Maladies inflammatoires chroniques intestinales, symptômes oculaires, inflammation oculaire

INTITULÉ ET ADRESSE :

UNIVERSITÉ DE LORRAINE

Faculté de Médecine de Nancy

9, avenue de la Forêt de Haye

54505 VANDOEUVRE LES NANCY Cedex 